\title{
ANÁLISE DA INFRA-ESTRUTURA EXISTENTE EM UNIDADES DE PRODUÇÃO AGRÍCOLA PARA PROCESSAMENTO DE MEL NA REGIÃO DO VALE DO PARAÍBA-SP ${ }^{1}$
}

\author{
Analysis of production unit infrastructure of honey processing agricultural in \\ the Paraiba Valley, São Paulo state - Brazil
}

Luiz Eugênio Veneziani Pasin², Mauro José Andrade Tereso ${ }^{3}$

\begin{abstract}
RESUMO
Objetivou-se neste trabalho analisar a existência de infra-estrutura para processamento de mel, em Unidades de Produção Agrícola-UPAs na região do Vale do Paraíba-SP. Para a realização desse trabalho utilizou-se o método de levantamento dos dados primários através de questionário. No período de janeiro de 2005 a março de 2006 realizou-se o trabalho com a amostra de 116 UPAs, que representam 33,7\% do total de unidades onde há atividade apícola. Para avaliar-se a associação e a relação de dependência entre as variáveis equipamentos, casa do mel, escolaridade, tempo na atividade e número de colméias na UPA, utilizou-se o teste do quiquadrado $X^{2}$. Os resultados revelaram que a centrífuga está presente em 58,6\% das UPAs e a casa do mel em 29,3\% das UPAs das unidades da região. O nível de escolaridade, tempo de permanência do apicultor na atividade e o número de colméias são variáveis que estabelecem relações significativas com a existência de centrífuga e de casa do mel na UPA.
\end{abstract}

Termos para indexação: Apicultura, centrífuga, casa do mel.

\section{ABSTRACT}

The aim of this paper is to analyze the infrastructure for honey process in Agricultural Production Units (UPAs) located in the Paraíba Valley (São Paulo state, Brazil). For this study to be carried out, the search of primary data method using a questionnaire was applied. From January 2005 to March 2006, 116 UPAs samples, representing 33.7\% of all bee-related UPAs were used. For the association and relation of dependence among the equipment variables, "casa do mel", staff education level, time in the activity and number of bee-hives in the UPA, the chi-square $\left(X^{2}\right)$ statistical tests were applied. The results revealed that centrifuges were present in 58.6\% of UPAs, while the type of "casa do mel" occured in $29.3 \%$ of UPAs in the region. The staff education level, time in the activity and number of bee-hives are variables which are important when considering the existence of centrifuges and "casa do mel" in the UPA.

Index terms: Bee-keeping, centrifuges, "casa do mel”.

(Recebido em 7 de maio de 2007 e aprovado em 3 de dezembro de 2007)

\section{INTRODUÇÃOO}

A região do Vale do Paraíba-SP desenvolve a atividade apícola há mais de um século e é conhecida no cenário da apicultura nacional pelos trabalhos científicos e serviços realizados pelos órgãos de pesquisa e centro de estudos existentes na região (APTA-Agência Paulista de Tecnologia dos Agronegócios, antigo Instituto de Zootecnia da Secretaria de Agricultura e Abastecimento do estado de São Paulo e CEA-Centro de Estudos Apícolas da Universidade de Taubaté). Em 2005 a produção de mel na região foi de 131.110 quilos, representando o equivalente a 6,0\% da produção total do Estado de São Paulo (IBGE, 2006).

O mel é um produto de origem animal e para sua comercialização é necessário que o apicultor atenda às exigências presentes na legislação ${ }^{4}$. Segundo Perosa et al. (2004), o alcance da competitividade do mel brasileiro devese basear no potencial das matas brasileiras aliado à produção de mel com qualidade.

A necessidade de se produzir mel de qualidade compatível com a determinação dos mercados mais exigentes é uma realidade, pois em março de 2006 a União Européia ${ }^{5}$ suspendeu as importações do mel brasileiro alegando exatamente a existência de falha no sistema de monitoramento da qualidade do mel brasileiro. Nesse caso Perez et al. (2006) e Souza (2006) salientaram a

4Portaria do MAPA, № 386, D O U 08/09/1997. Instrução Normativa do MAPA № 11, D.O.U., 23/10/2000. Instrução Normativa do MAPA № 22, D.O.U., 25/11/2005. Resolução da ANVISA RDC № 360, D.O.U., $26 / 12 / 2003$

5Jornal Oficial da União Européia, L 75/20, de 07 de março de 2006.

\footnotetext{
${ }_{1}^{1}$ Parte integrante da Tese de Doutorado do primeiro autor. Apoio financeiro PICD-UNITAU.

2Engenheiro Agrônomo, Doutor, Professor - Departamento de Ciências Agrárias/DPAGR - Universidade de Taubaté/UNITAU - Estrada Doutor José Luiz Cembranelli, 5000 - Bairro do Itaim - 12081-010 - Taubaté, SP - luiz.pasin@unitau.br

${ }^{3}$ Engenheiro Agrícola, Doutor, Professor - Faculdade de Engenharia Agrícola/FEAGRI - Universidade Estadual de Campinas/UNICAMP - Cidade

Universitária "Zeferino Vaz" Barão Geraldo - Cx. P. 6001 - 13083-970 - Campinas, SP - mauro@unicamp.br
} 
importância do setor apícola brasileiro se adequar aos padrões de exigência de qualidade, caso contrário corre o risco de ficar de fora do mercado internacional, por ausência de competitividade em relação à produção com qualidade.

Assim a adoção de boas práticas de higiene, o uso de equipamentos como centrífuga para extrair o mel, bem como a existência de um local adequado para o manuseio e extração de mel, a chamada casa do mel, são ações fundamentais e prioritárias exigidas por lei para a obtenção da qualidade do produto e a consequiente certificação de inspeção sanitária (BARRETO et al., 2006; COSTA et al., 2005).

Araújo et al. (2006) observaram que apicultores e comerciantes de mel demonstraram preocupação com o aumento da produtividade e comercialização, porém, não aplicam o mesmo empenho no exercício do controle de qualidade do mel durante a extração, beneficiamento e a venda. Entretanto, a atividade apícola deve visar sempre o alcance da excelência de qualidade em seus produtos, pois a competitividade do mel brasileiro está baseada, principalmente, no quesito qualidade e vários estudos têm demonstrado que a baixa qualidade em função da adulteração ao longo da cadeia produtiva e a oferta de produtos inadequados comprometem a abertura de novos mercados para esse produto (AZEREDO et al., 1999; KOMATSU et al., 2002; LEAL et al., 2001).

O Centro de Estudos Apícolas do Departamento de Ciências Agrárias da Universidade de Taubaté, em parceria com a Coordenadoria de Assistência Técnica Integral - CATI da Secretaria de Agricultura e Abastecimento do estado de São Paulo, constataram no ano de 2005, que a maior parte dos méis vendidos em estabelecimentos (comerciais) situados próximos e à margem da Rodovia Federal Presidente Eurico Gaspar Dutra $^{6}$ estavam fora dos padrões de identidade e qualidade (PIQ) do mel, conforme estabelece a legislação brasileira. Portanto, a oferta do produto mel certificado, de acordo com os parâmetros de qualidade, deve ser analisada como uma excelente oportunidade existente no mercado formal da região, em função do número de habitantes ${ }^{7}$ no Vale do Paraíba-SP em razão das pessoas que circulam, diariamente, nos estabelecimentos comerciais dessa importante Rodovia Federal.

Entretanto, é necessário que os agentes da cadeia produtiva apícola da região, principalmente o apicultor,

\footnotetext{
${ }^{6}$ Rodovia que atravessa toda a região do Vale do Paraíba-SP e estabelece a ligação entre a capitais dos Estados de São Paulo e Rio de Janeiro. ${ }^{7}$ O número de habitantes na região do Vale do Paraíba-SP é de 1.932 .581 (um milhão e novecentos e trinta e dois mil e quinhentos e oitenta e um) (IBGE, 2006).
}

tenham consciência da importância da adoção de boas práticas e controle de qualidade no processo produtivo do mel e a vigilância sanitária faça cumprir a lei nos estabelecimentos comerciais, coibindo o comércio do produto que se encontra fora do padrão.

Assim, esta pesquisa objetivou-se analisar a existência da infra-estrutura (Equipamentos e casa do mel) nas Unidades de Produção Agrícolas-UPAs que processam mel, na região do Vale do Paraíba-SP e a relação com as características intrínsecas do apicultor associados à escolaridade, tempo na atividade e número de colméias.

\section{MATERIAL E MÉTODOS}

O Vale do Paraíba-SP localiza-se no cone leste paulista do estado de São Paulo e faz divisa com os estados de Minas Gerais e Rio de Janeiro. Para realização desta pesquisa utilizou-se o método de levantamento dos dados, junto aos apicultores e técnicos da Casa Agricultura Municipal, com o uso de formulário Estruturado e Nãodisfarçado (BOYD \& WESTFALL, 1982). Para aplicação do formulário de pesquisa foram realizados 27 encontros com os técnicos das Casas da Agricultura Municipais e apicultores, no período de janeiro de 2005 a março de 2006.

Do total de 344 UPAs apícolas cadastradas, nas Casas de Agricultura Municipais e no Centro de Estudos Apícolas da Universidade de Taubaté, 116 foram pesquisadas representando $33,72 \%$ do total de unidades que desenvolvem a apicultura na região.

A escolha das UPAs ocorreu aleatoriamente e o número de amostras foi divido entre grupos de municípios (Tabela 1). A determinação desses grupos de municípios se justifica pela presença de apicultores cadastrados; pela logística operacional no trabalho de campo associado à proximidade entre os municípios e pelas características favoráveis ao desenvolvimento da apicultura em relação ao clima e vegetação.

$\mathrm{Na}$ análise estatística utilizou-se o teste do quiquadrado $X^{2}$ para o nível de significância $\mathrm{p}<0,05$ (Programa GraphPad Instat Version 3.00 Inc.), onde pesquisou-se a relação das variáveis, $X^{1}-$ Nível de escolaridade; $X^{2}$ - Tempo de permanência do apicultor na atividade; $X^{3}$ - Número de colméias existentes na UPA; $X^{4}$ - Centrífuga e $X^{5}$ - Casa do mel.

\section{RESULTADOS E DISCUSSÃO}

No Vale do Paraíba, entre as UPAs estudadas 97,0\% não possuem certificação da inspeção sanitária e mais de $90,0 \%$ comercializam toda sua produção no mercado informal. A apicultura na região apresenta-se como uma atividade de exploração, no setor rural, com possibilidades 
Tabela 1 - Grupo de municípios com o número de UPAs apícolas analisadas.

\begin{tabular}{|c|c|}
\hline Grupos de Municípios & $\begin{array}{c}\mathrm{N}^{0} \text { de UPAs } \\
\text { na amostra }\end{array}$ \\
\hline \multicolumn{2}{|l|}{ EDR* - Guaratinguetá } \\
\hline G.1. Areias, Bananal, Queluz e São José do Barreiro & 16 \\
\hline G.2. Cachoeira Paulista; Cruzeiro, Guaratinguetá; Lorena e Piquete & 15 \\
\hline G.3. Cunha e Lagoinha & 16 \\
\hline \multicolumn{2}{|l|}{ EDR ${ }^{*}$ - Pindamonhangaba } \\
\hline G.4. Caçapava, Pindamonhangaba, Taubaté e Tremembé & 12 \\
\hline G.5. Campos do Jordão, Monteiro Lobato, $\mathrm{St}^{\circ}$ A. do Pinhal e S. J. dos Campos & 17 \\
\hline G.6. Natividade da Serra; Redenção da Serra e São Luiz do Paraitinga & 19 \\
\hline G.7. Paraibuna & 21 \\
\hline Total & 116 \\
\hline
\end{tabular}

*Escritório de Desenvolvimento Regional da Secretaria de Agricultura e Abastecimento do Estado de São Paulo - EDR/SAA-SP.

de crescimento, e o aumento da produção de mel deverá ocorrer juntamente com o aumento da participação do apicultor no mercado formal. Esse crescimento de produção condicionado à inserção do apicultor no mercado formal estabelece um novo modelo de desenvolvimento para a apicultura familiar, pois a permanência do apicultor na informalidade certamente não permitirá que o setor apícola se desenvolva e promova alterações em seu atual "status quo".

O ingresso no mercado formal, bem como a própria saída da informalidade não é um ato espontâneo que deva ocorrer de maneira simples. Nesse caso, o desenvolvimento da apicultura na região deverá se basear em um modelo que possua como principal eixo condutor o entendimento do papel da apicultura na região, sua importância econômica como atividade geradora de renda e a própria potencialidade de crescimento, através da conquista do mercado formal existente no mercado consumidor local e regional.

Essa ação promoverá a ruptura do "círculo vicioso" que atualmente estimula a permanência do apicultor no mercado informal, onde o baixo nível de produção de mel no apiário ocorre em função das próprias dificuldades encontradas para escoar maiores volumes desse produto, uma vez que a capacidade de absorção do mercado informal é restrita e limitada.

Entre as UPAs estudadas $58,6 \%$ possuem centrífugas, sendo que nesse universo $93,0 \%$ possuem centrífugas do tipo manual. Essa característica evidencia a preocupação do apicultor em possuir o equipamento, porém, ajustando o tipo de equipamento com o próprio volume de produção e capacidade de investimento no apiário, uma vez que a centrífuga manual apresenta valor de mercado inferior à centrífuga elétrica.

Entre os apicultores que não possuem centrífugas prevalece a presença de duas ações estratégicas no processo de produção - utilizar a centrífuga de outra UPA ou improvisar métodos de trabalho que normalmente não são eficientes quanto à produção e higiene, e.g. extrair o mel dos favos com prensas e as mãos.

Ao relacionar o nível de escolaridade com a existência de centrífuga na UPA, a análise estatística apresentou associação significativa (Tabela 2). Ressaltase que, entre todos os apicultores que possuem centrífuga, $45,6 \%$ (31/68) tem curso superior, revelando que, na região, a existência desse equipamento ocorre com maior frequiência justamente nas UPAs em que os apicultores possuem maior escolaridade.

Ao relacionar o tempo de permanência do apicultor na atividade apícola com a existência da centrífuga na UPA, os resultados evidenciaram uma associação significativa. Nesse caso, a existência desse equipamento na UPA ocorre com maior frequiência justamente nos locais em que os apicultores desenvolvem a atividade apícola há mais tempo. Entre as UPAs em que o tempo do apicultor na atividade é superior a quatro anos $78,7 \%$ possuem centrífuga no apiário, nas UPAs em que o tempo do apicultor na atividade compreende de um a quatro anos, $70,4 \%$ possuem centrífuga e nas UPAs em que o tempo do apicultor na atividade é inferior a doze meses, somente $28,6 \%$ possuem centrífuga (Tabela 3 ). Portanto à medida que aumenta o tempo de permanência do apicultor na atividade apícola, as ações relacionadas à maior eficiência e qualidade na produção do mel, tornam-se mais freqüentes. 
Tabela 2 - Nível de escolaridade e a existência da centrífuga nas Unidades de Produção Agrícola, Vale do Paraíba-SP.

\begin{tabular}{|c|c|c|c|c|c|c|c|c|}
\hline \multirow{3}{*}{$\begin{array}{c}\text { Centrífuga } \\
\text { na } \\
\text { UPA }\end{array}$} & \multicolumn{6}{|c|}{ Nível de escolaridade } & & \\
\hline & \multicolumn{2}{|c|}{$\begin{array}{l}\text { Até ensino } \\
\text { fundamental }\end{array}$} & \multicolumn{2}{|c|}{$\begin{array}{l}\text { Ensino } \\
\text { médio }\end{array}$} & \multicolumn{2}{|c|}{$\begin{array}{l}\text { Ensino } \\
\text { superior }\end{array}$} & \multicolumn{2}{|c|}{ Total } \\
\hline & $\mathrm{N}$ & Freq. $(\%)$ & $\mathrm{N}$ & Freq. $(\%)$ & $\mathrm{N}$ & Freq. $(\%)$ & $\mathrm{N}$ & Freq.(\%) \\
\hline Possui & 12 & 57,1 & 25 & 46,3 & 31 & 75,6 & 68 & 58,62 \\
\hline Não Possui & 09 & 42,9 & 29 & 53,7 & 10 & 24,4 & 48 & 41,38 \\
\hline Total & 21 & 100,0 & 54 & 100,0 & 41 & 100,0 & 116 & 100,00 \\
\hline
\end{tabular}

O teste qui-quadrado indicou que há associação entre o nível de escolaridade e a existência de centrífuga no apiário $\left(X^{2}=8,279 ; p=\right.$ $0,0159)$

Tabela 3 - Tempo de permanência do apicultor na atividade apícola e existência da centrífuga nas Unidades de Produção Agrícola, Vale do Paraíba-SP.

\begin{tabular}{|c|c|c|c|c|c|c|c|c|}
\hline \multirow{3}{*}{$\begin{array}{c}\text { Centrífuga } \\
\text { na } \\
\text { UPA }\end{array}$} & \multicolumn{6}{|c|}{ Tempo do apicultor na atividade apícola } & \multirow{2}{*}{\multicolumn{2}{|c|}{ Total }} \\
\hline & \multicolumn{2}{|c|}{$0 \sim 12$ meses } & \multicolumn{2}{|c|}{$1 \sim 4$ anos } & \multicolumn{2}{|c|}{$>4$ anos } & & \\
\hline & $\mathrm{N}$ & Freq. $(\%)$ & $\mathrm{N}$ & Freq. $(\%)$ & $\mathrm{N}$ & Freq. $(\%)$ & $\mathrm{N}$ & Freq. $(\%)$ \\
\hline Possui & 12 & 28,6 & 19 & 70,4 & 37 & 78,7 & 68 & 58,6 \\
\hline Não Possui & 30 & 71,4 & 08 & 29,6 & 10 & 22,3 & 48 & 41,4 \\
\hline Total & 42 & 100,0 & 27 & 100,0 & 47 & 100,0 & 116 & 100,0 \\
\hline
\end{tabular}

O teste qui-quadrado indicou que há associação entre o tempo do apicultor na atividade apícola e a existência de centrífuga na UPA. $\left(X^{2}=25,001 ; p<0,0001\right)$.

Em relação ao número de colméias presente na UPA, os resultados demonstraram que existe uma associação entre a quantidade de colméias existentes nas UPAs e a presença da centrífuga (Tabela 4). A existência do equipamento que envolve tecnologia e higiene, ocorreu com maior frequiência nas UPAs que possuem maior número de colméias. Silva (2000) mencionou que o número de colméias presentes no apiário determina a tipologia de classificação ${ }^{8}$ do apiário, relacionando que a quantidade de colméias é um dos principais fatores associados à produção, principalmente sobre a tecnologia e a mão-deobra empregada.

Entre as UPAs com tipologia-profissional 92,3\% possuem centrífuga; entre aquelas com tipologiafamiliar profissional 83,3\% possuem centrífuga e entre as UPAs com tipologia-familiar somente $42,5 \%$ possuem esse equipamento (Tabela 4). Portanto, a ausência de centrífuga para extração de mel aparece com maior frequiência nas UPAs classificadas com tiplogia-familiar, revelando que o

${ }^{8}$ Apiário Familiar = Até 20 colméias; Apiário Familiar Profisional $=21$ até 50 colméias; Apiário Profissional $=51$ até 200 colméias; Apiário Profissional $\sim$ Empresarial $=201$ até 500 colméias; Apiário $=$ Empresarial $=$ Acima de 500 colméias (SILVA, 2000). número de colméias existentes nesses locais também poderá ser considerado como um dos fatores limitantes para existência do equipamento.

A ausência de uma casa do mel na UPA ocorre em 70,7\% das unidades da região e segundo apicultores entrevistados, a utilização desse local, em caráter de empréstimo ou parceria, é baixa. Essa situação revela que a maior parte dos apicultores improvisa um local de trabalho na própria residência para manusear e extrair o mel.

A manipulação dos produtos apícolas deve sempre ocorrer em ambiente apropriado com a infra-estrutura adequada (BARRETO et al., 2006). Entretanto, no Vale do Paraíba-SP somente 1/3 das UPAs estudadas possuem o local adequado, evidenciando a necessidade da implantação de um programa regional que promova o alcance da qualidade desses produtos.

Ao relacionar o nível de escolaridade com a existência da casa do mel na UPA, constatou-se a existência de uma associação significativa (Tabela 5). Nesse caso, a infra-estrutura, que proporciona o manuseio em local adequado, encontra-se relacionada com a escolaridade do apicultor da região. Ela foi proporcionalmente maior entre os apicultores que 
Tabela 4 - O número de colméias no apiário e a existência da centrífuga nas Unidades de Produção Agrícola, Vale do Paraíba-SP.

\begin{tabular}{|c|c|c|c|c|c|c|c|c|}
\hline \multirow{3}{*}{$\begin{array}{c}\text { Centrífuga } \\
\text { na } \\
\text { UPA }\end{array}$} & \multicolumn{6}{|c|}{ Número de colméias } & & \\
\hline & \multicolumn{2}{|c|}{$\begin{array}{l}\text { Familiar } \\
\text { (até 20) }\end{array}$} & \multicolumn{2}{|c|}{$\begin{array}{c}\text { Familiar profissional } \\
\text { (entre } 21 \text { até } 50 \text { ) }\end{array}$} & \multicolumn{2}{|c|}{$\begin{array}{c}\text { Profissional } \\
\text { (entre } 51 \text { até 200) }\end{array}$} & \multicolumn{2}{|c|}{ Total } \\
\hline & $\mathrm{N}$ & Freq. $(\%)$ & $\mathrm{N}$ & Freq. $(\%)$ & $\mathrm{N}$ & Freq. $(\%)$ & $\mathrm{N}$ & Freq. $(\%)$ \\
\hline Possui & 31 & 42,5 & 25 & 83,3 & 12 & 92,3 & 68 & 58,6 \\
\hline Não Possui & 42 & 57,5 & 05 & 16,7 & 01 & 7,7 & 48 & 41,4 \\
\hline Total & 73 & 100,0 & 30 & 100,0 & 13 & 100,0 & 116 & 100,0 \\
\hline
\end{tabular}

Tabela 5 - O nível de escolaridade e a existência da casa do mel nas Unidades de Produção Agrícola, Vale do ParaíbaSP.

\begin{tabular}{|c|c|c|c|c|c|c|c|c|}
\hline \multirow{4}{*}{$\begin{array}{c}\text { Casa do } \\
\text { mel na } \\
\text { UPA }\end{array}$} & \multicolumn{6}{|c|}{ Nível de Escolaridade } & & \\
\hline & \multirow{2}{*}{\multicolumn{2}{|c|}{$\begin{array}{c}\text { Até } \\
\text { Fundamental }\end{array}$}} & \multirow{2}{*}{\multicolumn{2}{|c|}{$\begin{array}{l}\text { Ensino } \\
\text { Médio }\end{array}$}} & \multirow{2}{*}{\multicolumn{2}{|c|}{$\begin{array}{l}\text { Ensino } \\
\text { Superior }\end{array}$}} & \multirow{2}{*}{\multicolumn{2}{|c|}{ Total }} \\
\hline & & & & & & & & \\
\hline & $\mathrm{N}$ & Freq. $(\%)$ & $\mathrm{N}$ & Freq. $(\%)$ & $\mathrm{N}$ & Freq. $(\%)$ & $\mathrm{N}$ & Freq. $(\%)$ \\
\hline Possui & 03 & 14,2 & 10 & 18,5 & 21 & 51,2 & 34 & 29,3 \\
\hline Não Possui & 18 & 85,9 & 44 & 81,5 & 20 & 48,8 & 82 & 70,7 \\
\hline Total & 21 & 100,0 & 54 & 100,0 & 41 & 100,0 & 116 & 100,0 \\
\hline
\end{tabular}

O teste qui-quadrado indicou que há associação entre o nível de escolaridade e a existência de casa do mel na UPA $\left(X^{2}=14,822\right.$; $p=0,0006)$.

possuem maior escolaridade, sendo que $61,8 \%(21 / 34)$ das UPAs avaliadas e que possuem casa do mel, a escolaridade do apicultor é de ensino superior.

Ao relacionar o tempo de permanência na atividade apícola com a existência de casa do mel na UPA, constatouse uma associação significativa. Portanto, a presença de infraestrutura adequada ocorre proporcionalmente com maior freqüência nas unidades em que os apicultores já desenvolvem a atividade apícola por mais tempo. Naquelas em que o tempo do apicultor na atividade é superior a quatro anos, $42,5 \%$ possuem casa do mel. Entre as UPAs em que o tempo do apicultor na atividade compreende entre um e quatro anos $29,6 \%$ possuem casa do mel e nas unidades em que o tempo do apicultor na atividade apícola é inferior a 12 meses, somente 14,3\% possuem essa infra-estrutura (Tabela 6).

No Vale do Paraíba-SP, a maior parte dos apicultores inicia a atividade apícola com uma infra-estrutura mínima e as providências para instalação e manuseio em local adequado, não ocorre necessariamente no início do desenvolvimento da atividade na UPA.
Em relação ao número de colméias no apiário, os resultados revelaram que existe uma associação significativa entre a quantidade de colméias presentes nas UPAs e a existência de casa do mel. Essa infra-estrutura ocorre com maior frequiência nas unidades que possuem o maior número de colméias, e entre as aquelas com tipologiafamiliar somente 19,2\% possuem casa do mel. Já entre as UPAs com tipologia-profissional e tipologiafamiliar profissional, aproximadamente, $46,0 \%$ possuem casa do mel (Tabela 7).

A justificativa dos apicultores da região, que não possuem centrífuga ou casa do mel, está pautada na necessidade do investimento e por causa da baixa produção de mel existente na UPA, esse apicultor não é estimulado a adquirir a centrífuga e implantar a casa do mel. Assim, a produção de mel com higiene e qualidade conforme recomenda os órgãos governamentais, depende de ações estratégicas e parcerias entre os apicultores, onde o eixo dessa ação possivelmente se sustentaria na criação de associações e cooperativas. 
Tabela 6 - Tempo permanência na atividade apícola e existência de casa do mel nas Unidades de Produção Agrícola, Vale do Paraíba-SP.

\begin{tabular}{|c|c|c|c|c|c|c|c|c|}
\hline \multirow{3}{*}{$\begin{array}{c}\text { Casa do } \\
\text { mel na } \\
\text { UPA }\end{array}$} & \multicolumn{6}{|c|}{ Tempo na atividade apícola } & \multirow{2}{*}{\multicolumn{2}{|c|}{ Total }} \\
\hline & \multicolumn{2}{|c|}{$0 \sim 12$ meses } & \multicolumn{2}{|c|}{$1 \sim 4$ anos } & \multicolumn{2}{|c|}{$>4$ anos } & & \\
\hline & $\mathrm{N}$ & Freq. $(\%)$ & $\mathrm{N}$ & Freq. $(\%)$ & $\mathrm{N}$ & Freq. $(\%)$ & $\mathrm{N}$ & Freq. $(\%)$ \\
\hline Possui & 06 & 14,3 & 8 & 29,6 & 20 & 42,5 & 34 & 29,3 \\
\hline Não Possui & 36 & 85,7 & 19 & 70,4 & 27 & 57,5 & 82 & 70,7 \\
\hline Total & 42 & 100,0 & 27 & 100,0 & 47 & 100,0 & 116 & 100,0 \\
\hline
\end{tabular}

O teste qui-quadrado indicou que há associação entre o tempo na atividade apícola e a existência de casa do mel na UPA. $\left(X^{2}=8,555\right.$; $p=0,0139$ ).

Tabela 7 - O número de colméias no apiário e existência de casa do mel nas Unidades de Produção Agrícola, Vale do Paraíba-SP.

\begin{tabular}{|c|c|c|c|c|c|c|c|c|}
\hline \multirow{3}{*}{$\begin{array}{c}\text { Casa do } \\
\text { mel na } \\
\text { UPA }\end{array}$} & \multicolumn{6}{|c|}{ Número de Colméias } & & \\
\hline & \multicolumn{2}{|c|}{$\begin{array}{l}\text { Familiar } \\
\text { (até 20) }\end{array}$} & \multicolumn{2}{|c|}{$\begin{array}{c}\text { Familiar Profissional } \\
\text { (entre } 21 \text { até } 50)\end{array}$} & \multicolumn{2}{|c|}{$\begin{array}{c}\text { Profissional } \\
\text { (entre } 51 \text { até 200) }\end{array}$} & \multicolumn{2}{|c|}{ Total } \\
\hline & $\mathrm{N}$ & Freq. $(\%)$ & $\mathrm{N}$ & Freq. $(\%)$ & $\mathrm{N}$ & Freq. $(\%)$ & $\mathrm{N}$ & Freq. $(\%)$ \\
\hline Possui & 14 & 19,2 & 14 & 46,7 & 06 & 46,2 & 34 & 29,31 \\
\hline Não Possui & 59 & 80,8 & 16 & 53,3 & 07 & 53,8 & 82 & 70,69 \\
\hline Total & 73 & 100,0 & 30 & 100,0 & 13 & 100,0 & 116 & 100,00 \\
\hline
\end{tabular}

O teste qui-quadrado indicou que há associação entre número de colméias e a existência de centrífuga na UPA. $\left(X^{2}=9,759\right.$; $p=0,0076)$.

\section{CONCLUSÕES}

Na região do Vale do Paraíba-SP, o nível de escolaridade do apicultor, tempo de permanência do apicultor e o número de colméias existentes nas UPAs são variáveis que influenciam e estão diretamente associadas à presença de tecnologia na Unidade de Produção Agrícola. Essas variáveis estabeleceram relações significativas com a existência de centrífuga e de casa do mel entre as UPAs amostradas.

\section{REFERÊNCIAS BIBLIOGRÁFICAS}

ARAÚJO, D. R. de; SILVA, R. H. D. da; SOUSA, J. dos S. Avaliação da qualidade físico-química do mel comercializado na cidade de Crato, CE. Revista de Biologia e Ciências da Terra, Campina Grande, v. 6, n. 1, p. 51-55, set. 2006.

AZEREDO, M. A. A.; AZEREDO, L. da C.; DAMASCENO, J. G. Características físico-químicas dos méis do município de São Fidélis-RJ. Ciência e Tecnologia de Alimentos, Campinas, v. 19, n. 1, p. 3-7, 1999.
BARRETO, L. M. R. C.; PEÃO, G. F. R.; DIB, A. P. da S. Higienização e sanitização na produção apícola. Taubaté: Cabral Editora, 2006. 137 p.

BOYD, H. M.; WESTFALL, R. Pesquisa mercadológica e casos. Rio de Janeiro: FGV, 1982. 384 p.

COSTA, C. C. da; FERREIRA, R. G.; PRATA FILHO, D. de A. A influência de centrífuga no processamento de mel de abelha. Engenharia Agrícola, Jaboticabal, v. 25, n. 3, p. 809-816, set./dez. 2005.

INSTITUTO BRASILEIRO DE GEOGRAFIA E ESTATISTICA. IBGE. 2006. <www.ibge.gov.br>. Acesso em: 25 jan. 2007.

KOMATSU, S. S.; MARCHINI, L. C.; MORETI, A. C. de C. C. Análises físico-químicas de amostras de méis de flores silvestres, de eucalipto e de laranjeira, produzidos por Apis mellifera L., 1758 (Hymenoptera, Apidae) no Estado de São Paulo: 2. conteúdo de açúcares e de proteína. Ciência e Tecnolologia de Alimentos, Campinas, v. 22, n. 2, p. 143146, maio/ago. 2002. 
LEAL, V. M.; SILVA, M. H.; JESUS, N. M. Aspectos físicoquímico do mel de abelhas comercializado no município de Salvador-Bahia. Revista Brasileira de Saúde e Produção Animal, Salvador, v. 1, n. 1, p. 14-18, 2001.

PEREZ, L. H.; RESENDE, J. V. de; FREITAS, B. B. de. Mel: câmbio e embargo europeu podem prejudicar exportações em 2006. Análises e Indicadores do Agronegócio, São Paulo, v. 1, n. 4, abr. 2006. Disponível em: <http://www.iea.sp.gov.br/ out/verTexto.php?codTexto=5209>. Acesso em: 6 maio 2006.
PEROSA, J. M. Y.; ARAUCO, E. M. R.; SANTOS, M. L. de A.; ALBARRACIN, V. N. Parâmetros de competitividade do mel brasileiro. Revista Informações Econômicas, São Paulo, v. 34, n. 3, mar. 2004.

SILVA, W. P. da. Manual de comercialização apícola. Maceió: Sebrae, 2000.

SOUZA, D. C. A profissionalização da apicultura no Brasil. Revista Sebrae Agronegócios, Brasília, n. 3, p. 50-51, maio 2006. 